\title{
Corona Virus Disease-2019 (COVID-19) - An Insight on Treatment Options and Prevention Protocol
}

\section{Haben Fesseha, MVSc, DVM ${ }^{1, *}$ and Yohannes Fesseha, MD $^{2}$}

${ }^{1}$ Department of Veterinary Surgery and Diagnostic Imaging, School of Veterinary Medicine, Wolaita Sodo University, P. O. Box 138, Wolaita Sodo,Ethiopia

${ }^{2}$ College of Health Science, School of Medicine, Mekelle University, P.O. Box:1871, Mekelle, Ethiopia

*Corresponding author: Haben Fesseha, Department of Veterinary Surgery and Diagnostic Imaging, School of Veterinary Medicine, Wolaita SodoUniversity, P. O. Box 138, Wolaita Sodo, Ethiopia.

Received date: 15 May 2020; Accepted date: 05 June 2020; Published date: 10 June 2020

Citation: Fesseha H, Fesseha Y (2020) Corona Virus Disease-2019 (COVID-19) - An Insight on Treatment Options and Prevention Protocol. J CommMed Pub Health Rep 1(1): https://doi.org/10.38207/jcmphr20208

Copyright: (C) 2020 Fesseha H. This is an open-access article distributed under the terms of the Creative Commons Attribution License, which permitsunrestricted use, distribution, and reproduction in any medium, provided the original author and source are credited.

\begin{abstract}
Many coronaviruses causing a variety of pathological disease in animals and maintained their cycle in nature and causing zoonotic infections, allowingthem for genetic recombination, resulting in novel viruses. COVID-19 is the third coronavirus to emerge in the human population in the past 18 years after Severe Acute Respiratory Syndrome Coronavirus (SARS-CoV) is an outbreak in 2002 and the Middle East Respiratory Syndrome Coronavirus (MERS-CoV) outbreak in 2012. Severe acute respiratory syndrome coronavirus 2 (SARS-CoV-2, previously known as novel coronavirus or 2019- nCoVis the sixth emerging viral zoonotic disease from China at the end of 2019 and it is a threat to a million of people across the world and a reason for deathof hundred thousand of peoples across the globe. Based on evidence, it has mean incubation period of 6.4 days and transmitted mainly through microdroplets and contact with infected person. Fever, Cough, dyspnea, and fatigue are the most common symptom of the patients. Reports also indicatedthat underlying diseases such as cardiovascular disease, hypertension, and diabetes mellitus further precipitating the condition and more mortality caseswere recorded. Even though there is no effective treatment against COVID-19, different antibiotic, antiviral (nucleoside analogues, neuraminidase inhibitors, DNA synthesis inhibitors and chloroquine) have been proposed as a potential drug options for COVID-19. Control measures are the best option preventing the spread of the infection and reduced the incidence of new cases. Therefore, close monitoring of the situation and a coordinated global response is desperately needed to prepare health systems to meet this unprecedented challenge.
\end{abstract}

Keywords: COVID-19, Prevention, SARS-CoV, Treatment

\section{Introduction}

Emerging and reemerging pathogens are global challenges for public health. Coronavirus disease 2019 (COVID-19), a form of respiratory andsystemic zoonosis caused by a virus CORONA (Latin Crown, from the structure of the virus under electron microscope) belonging to the Coronaviridae family [1-3]. The new Corona virus (COVID19) identified as the cause of the acute respiratory disease in humans since the end of December 2019(2019-nCoV), later labeled as SARS CoV2 byWorld Health Organization is a different strain of Corona virus from SARS and MERS CORONA viruses. The difference is not limited to genetic make-up only but also in the clinical presentations case fatality and the rate of spread across the globe. The recen pandemic is a great global challenge causing severe acute respiratory infection and remains one of the leading causes of mortality around the world [4].

Coronaviruses are enveloped non-segmented positive-sense RNA viruses belonging to the family Coronaviridae and the order Nidoviralesand broadly distributed in humans and other mammals. A novel coronavirus $(\mathrm{nCoV})$ is a new strain that has not been previously identified in humans $[\mathbf{1}, \mathbf{5}, \mathbf{6}]$. Most people who fall sick with COVID- 19will experience mild to moderate symptoms and recover without special treatment. The virus that causes COVID-19 is mainly transmitted through droplets generated when an infected person coughs, sneezes, orexhales. These droplets are too heavy to hang in the air, and quickly fallon floors or surfaces. Besides, a person can be infected by breathing in the virus if you are within close proximity of someone who has COVID-19, or by touching a contaminated surface and then your eyes, nose or mouth [2,7].

Moreover, the spread of coronavirus disease 2019 (COVID-19) is becoming unstoppable and has already reached the necessary epidemiological criteria for it to be declared a pandemic. According to the 115-Situation Report of the World Health Organization (WHO), thedisease has already involved all continents, with over 4,248,389 confirmed cases in 197 different countries, and nearly 294,046 deaths until May 14, 2020 [8]. The COVID 19 pandemic is causing huge stresson the health care system of all countries in the world. The impact of thepandemic is both social and economic. Thus, the pandemic needs an early intervention with optimal global commitment and communitymobilization help to flatten the curve 
averting occurrence of many casesand deaths [9]

The pandemic started when the world was not ready even for commonly known epidemics and when mobility for business and tourism was highand very simple due to globalization. Poor hand hygiene practice, overcrowding, and close physical contacts like hand shaking contributesfor the fast spread of the virus with in very short period. Scarcity of supplies for hand hygiene and lack of PPE in most health facilities are good evidences for our unpreparedness for an infectious disease pandemic of this scale [10].

The globe community should wisely use the limited resources of the world such as trained human and material resources as the case in most part of the pandemic areas. Thus, these limited resources should be allocated wisely to prevent the transmission of the disease. Besides, implementation of a uniform and evidence-based preventive and treatment protocol at all levels of health care system is considered as thebetter recommendation by World health organization \& CDC. Generally,this helps to treat and give priority for most affected areas and to reduces or limit further spread of the infection in that locality as well as other part of the country $[\mathbf{2 , 1 1}]$.

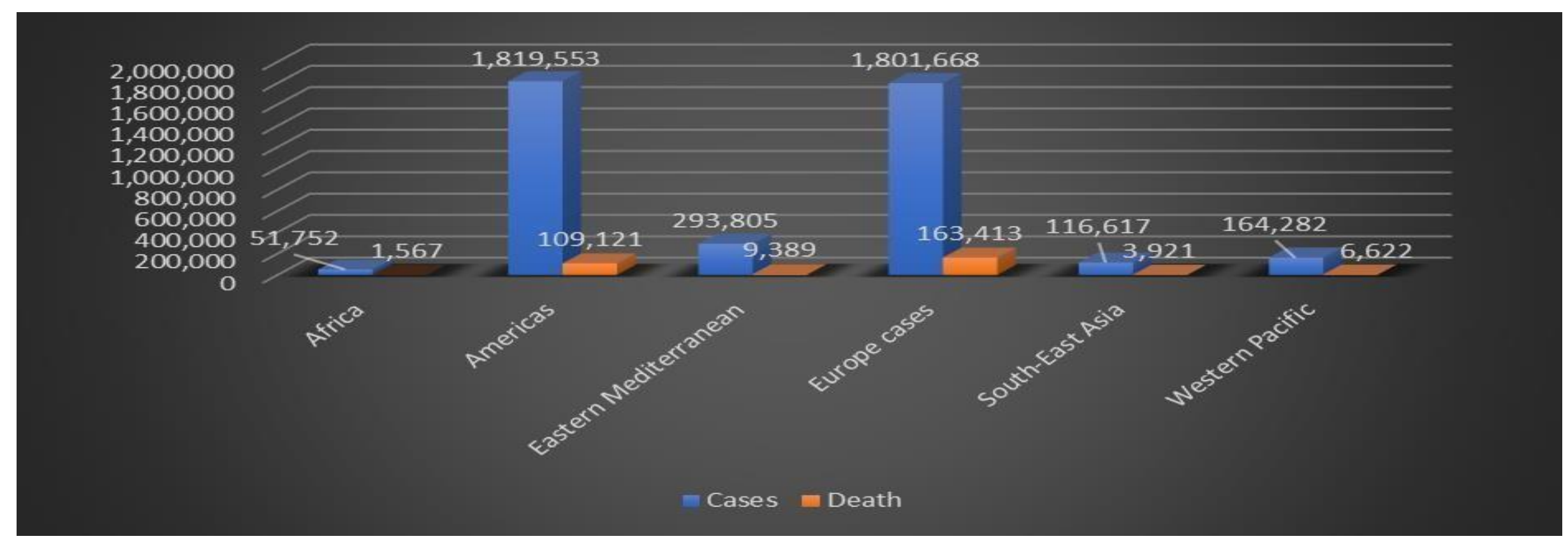

Table 1: The status of COVID-19 on the globe, Source: WHO situation report May 15, 2020.

\section{Etiology, Transmission and Predisposing factors}

Coronavirus disease-19 (COVID-19) is cause by Coronavirus, which is large, mostly spherical, sometimes pleomorphic (changeable in shape), particles with bulbous surface projections. The average diameter of the virus particles is around $125 \mathrm{~nm}(0.125 \mu \mathrm{m})$. The diameter of theenvelope is $85 \mathrm{~nm}$ and the spikes are $20 \mathrm{~nm}$ long. The envelope of the virus in electron micrographs appears as a distinct pair of electrons- dense shells that are relatively opaque to the electron beam used to scan the virus particle. The viral envelope consists of a lipid bilayer, in whichthe membrane (M), envelope (E) and spike (S) structural proteins are anchored. The ratio of E:S:M in the lipid bilayer is approximately1:20:300. On average a coronavirus particle has 74 surface spikes. A subset of coronaviruses (specifically the members of beta coronavirus subgroup A) also have a shorter spikelike surface protein called hemagglutinin esterase (HE) [5,12,13].
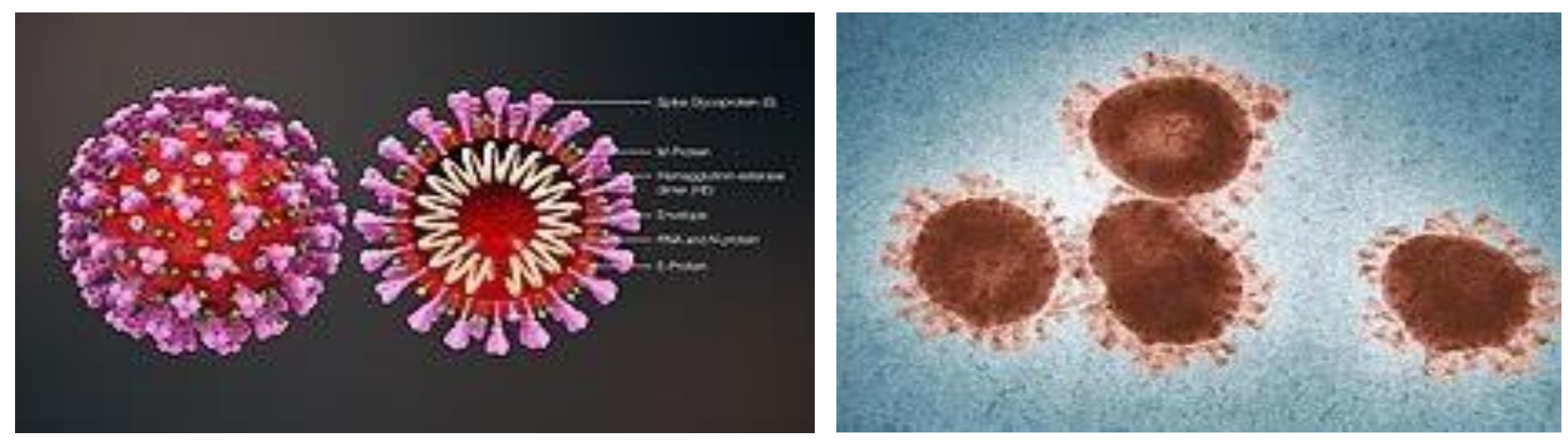

\section{Figure 2: Cross-sectional model of a coronavirus}

\section{Source:[14]}

Coronavirus is one of the pathogens that causes respiratory tract infection in human. The four other human coronaviruses (HCoVOC43,HCoV-229E, HCoV-NL63, HCoV-HKU1) induce mild upper respiratory disease like common cold. Whereas there are three human coronaviruses that produce potentially severe symptoms including Middle East respiratory syndrome-related coronavirus (MERS-CoV), 
$\beta-\mathrm{CoV}$, Severe acute respiratory syndrome coronavirus (SARS-CoV), $\beta-\mathrm{CoV}$, and severe acute respiratory syndrome coronavirus 2 (SARSCoV-2), $\beta-\operatorname{CoV}[\mathbf{1 5}, 16]$.

Many domestic and wild animals, including camels, cattle, cats, and bats, may serve as hosts for coronaviruses [17]. Droplet from infected person during cough, handshake, overcrowding and sneezing and contaminatedsurfaces (inanimate objects such as tables, desks) are the main source and are possible means of transmission for the disease. Besides, infectedcarriers can shed viruses into the environment [2,18]. Sputum and saliva carry large amounts of virus. Although Pathogenesis

COVID-19 is not a sexually transmitted infection, kissing, intimate contact, and faecal-oral routes are suspected to transmit the virus. Somemedical procedures are aerosol-generating and result in the virus being transmitted more easily than normal [12].

Human coronaviruses infect the epithelial cells of the respiratory tract, while animal coronaviruses generally infect the epithelial cells of the digestive tract. SARS coronavirus, for example, infects via an aerosol route, the human epithelial cells of the lungs by binding to the angiotensin-converting enzyme 2 (ACE2) receptor. Transmissible gastroenteritis coronavirus (TGEV) infects, via a fecal-oral route, the pigepithelial cells of the digestive tract by binding to the alanine aminopeptidase (APN) receptor [18, 19]. Based on different literatures, Immunocompromised individuals, old age peoplesaffected with diseases having malignancy and Patients chronic disorders including bronchial asthma, diabetes and cardiovascular disorder are the most susceptible peoples and are at risk of getting the infection with this deadly virus $[\mathbf{1 8 , 1 9 ]}$.

COVID-19 has unique feature as it causes both upper and lower respiratory infections. Moreover, few data are available about microscopic lesions and the pathophysiology of COVID-19 [20]. Thisdiagram below illustrates the possible pathogenesis of the disease.

\section{是 \\ The virus attaches to specific cellular receptors (upper \& lower RT) via the spike protein

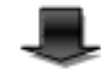 \\ Transformational change, leading to fusion between the viral and the host cell membrane \\ Release of nucleocapsid into the cell (transcription and translation) \\ 旦 \\ Alteration of DNA and production of protein and certain specific enzymes

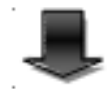 \\ Alteration of cell function and production

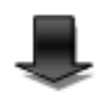 \\ Release of excess cytokines and chemokines (IL-1 $\beta$, IL- 6 , IL-7, IL-8, IL-9, IL-10 \& TNF- $\alpha$ )}

Hyperinflammation is the main cause of death due to complication/ effects caused by "cytokine storm syndrome". As a result, Severe acute respiratory distress syndrome (SARS) and Fulminant myocarditis (cardiac affinity) Furthermore, the main gross pathological findings at autopsy are pleurisy, pericarditis, lung consolidation and pulmonary edema. Besides,four type of pneumonia can be observed including minor pneumonia (minor serous exudation, minor fibrin exudation), mild pneumonia (pulmonary edema, pneumocyte hyperplasia, large atypical pneumocytes, interstitial

\section{Clinical signs}

The incubation period for COVID-19 is typically five to six days but may range from two to 14 days, although $97.5 \%$ of people who develop symptoms will do so within 11.5 days of infection [8,22]. The two highly pathogenic viruses that emerged previously, the severe acute respiratorysyndrome coronavirus (SARS-CoV) and the Middle East respiratory syndrome coronavirus (MERS-CoV) cause more severe, pneumonialikesymptoms, severe acute respiratory syndrome inflammation with lymphocytic infiltration andmultinucleated giant cell formation), severe pneumonia (diffuse alveolardamage (DAD) with diffuse alveolar exudates. DAD is the cause of acuterespiratory distress syndrome (ARDS) and severe hypoxemia) and healing pneumonia (organization of exudates in alveolar cavities and pulmonary interstitial fibrosis) [21]. Additionally, disseminated intravascular coagulation (DIC), leukoerythroblastic reaction and microvesicular steatosis in liver can also be seen in infected patients $[14,20]$.

(SARS) and sometimes fatality in humans [15].

According to recent evidence and experience, the signs and symptoms ofCOVID-19 may appear two to 14 days after exposure and ranged from mild symptoms to severe illness and death. Fever is the most common symptom, although some older people and those with other healthproblems experience fever later in the disease. In one study, $44 \%$ of people had fever when they presented to the hospital, while 
$89 \%$ went on to develop fever at some point during their hospitalization. A lack offever does not verify someone is disease free $[23,24]$.

Moreover, non-productive cough, loss of appetite, sore throat, aches, runny nose, dyspnea, myalgia, fatigue, shortness of breath, sputum production, normal or decreased leukocyte counts, and radiographic evidence of pneumonia are some of the observed clinical manifestations.Symptoms such as nausea, vomiting, and diarrhea have been observed in varying percentages. Less common symptoms include sneezing, runny nose, or sore throat [22]. In more severe cases, the infection can cause pneumonia, severe acute respiratory syndrome, shock, organ dysfunction (acute cardiac injury, kidney failure), and even death [7]. However, a minority of cases do not develop noticeable symptoms at any point in time. These

\section{Case definitions for COVID-19}

According to World Health Organization and Center for Disease controland prevention, the different patients of this infection cases are classifiedbased on the clinical manifestation of the disease.

Suspected case: A person having fever $\left(>38^{\circ} \mathrm{C}\right)$ or history of fever and symptoms of respiratory tract illness and close contact with a person whois under investigation or confirmed for COVID-19. Also, a person with cough, difficulty in breathing and a history of travel to or residence in a country/area or territory reporting local transmission of COVID-19 disease during the 14 days prior to symptom onset.

Confirmed case: this is when a person is confirmed of COVID-19 infection with laboratory irrespective of clinical signs and symptoms. Uncomplicated illness: Patients with uncomplicated upper respiratory tract viral infection, may have nonspecific symptoms such as fever, cough, sore throat, nasal congestion, malaise, headache, muscle pain or malaise. The elderly and immunosuppressed may present with atypical symptoms. These patients may be asymptomatic that is do not have anysigns of dehydration, sepsis, or shortness of breath. Symptomatic cases of COVID-19 have different Clinical syndromes andcategorized into the following groups:

Mild Pneumonia: Patient with pneumonia and no signs of severe

\section{Diagnostic approaches}

The World Health Organization (WHO) has published several testing protocols for the disease [29]. The standard method of testing is realtime reverse transcription polymerase chain reaction (rRT-PCR). The test is typically done on respiratory samples obtained by a nasopharyngeal swab; however, a nasal swab or sputum sample may alsobe used. Results are generally available within a few hours to two days $[\mathbf{1 4 , 3 0 ]}$. Blood tests can be used, but these require two blood samples taken two weeks apart, and the results have little immediate value [10].

Along with laboratory testing, chest CT scans may be helpful to diagnoseCOVID-19 in individuals with a high clinical suspicion of asymptomatic carriers tend not to get tested, and their role in transmission is not yet fully known.

However, preliminary evidence suggests they may contribute to the spread of the disease [25]. Complications may include pneumonia, acute respiratory distress syndrome (ARDS), multi-organ failure, septic shock, and death. Cardiovascular complications may include heart failure, arrhythmias, heart inflammation, and blood clots [24,26]. Approximately $20-30 \%$ of people who present with COVID-19 have elevated liver enzymes reflecting liver injury. Neurologic manifestations include seizure, stroke, encephalitis, and Guillain-Barré syndrome [27]. Following the infection,children may develop pediatric multisystem inflammatory syndrome, which has symptoms like Kawasaki disease, and die [28].

pneumonia. Child with non-severe pneumonia has cough or difficulty breathing with fast breathing.

Severe Pneumonia (Adolescent or Adult): Fever or suspected respiratory infection, plus one of respiratory rate $>30$ breaths $/ \mathrm{min}$, severe respiratory distress, or $\mathrm{SpO}_{2}<90 \%$

Acute Respiratory Distress Syndrome: Onset-new or worsening respiratory symptoms within 1 week of known clinical insult. Chest imaging (radiograph, CT scan, or lung ultrasound): bilateral opacities, not fully explained by effusions, lobar or lung collapse, or nodules. Origin of edema-respiratory failure not fully explained by cardiac failure or fluid overload. Objective assessment (e.g. echocardiography) is needed to exclude hydrostatic cause of edema if no risk factor is present.

Sepsis: Adults-life-threatening organ dysfunction caused by a dysregulated host response to suspected or proven infection, with organdysfunction.

Septic Shock: Adults-persisting hypotension despite volume resuscitation, requiring vasopressors to maintain Mean Arterial Pressure $(\mathrm{MAP}) \geq 65 \mathrm{mmHg}$ and serum lactate level $>2 \mathrm{mmol} / \mathrm{L}$.

infection but are not recommended for routine screening $[2,15]$. Bilateral multilobar ground-glass opacities with a peripheral, asymmetric, and posteriordistribution are common in early infection. Subpleural dominance, crazypaving (lobular septal thickening with variable alveolar filling), and consolidation may appear as the disease progresses [17,31].

Chinese scientists were able to isolate a strain of the coronavirus and publish the genetic sequence so laboratories across the world could independently develop polymerase chain reaction (PCR) tests to detect infection by the virus [30,32,33]. As of 4 April 2020, antibody tests (which may detect active infections and whether a person had 
been infected in the past) were in development, but not yet widely used. The Chinese experience with testing has shown the accuracy is only 60 to $70 \%$. The FDA in the United States approved the first point-of-care teston 21 March 2020 for use at the end of that month [12].

Different screening and confirmatory tests are conducted on suspected patient to confirm the case. According to research reports of different authors, there are different enzymatic, blood and multiorgan pathologiesand abnormalities are observed on the patient upon laboratoryinvestigation. Among these, decreased lymphocytes count

\section{Prevention}

Preventive measures to reduce the chances of infection include staying at home, avoiding crowded places, keeping distance from others, washing hands with soap and water often and for at least 20 seconds, practicing good respiratory hygiene, and avoiding touching the eyes, nose, or mouth with unwashed hands [34]. The Centers for Disease Control and Prevention (CDC) of the USA recommends covering the mouth and nose with a tissue when coughing or sneezing and recommends using the inside of the elbow if no tissue is available $[12,35]$.

According to the WHO and CDC, the use of masks is recommended onlyif a person is coughing or sneezing or when one is taking care of someone with a suspected infection. Also, the use of cloth face coverings is recommended in public settings where other social distancing measures are difficult to maintain, in part to limit transmission by asymptomatic individuals. Several countries have recommended that healthy individuals wear face masks or cloth face coverings (like scarves or bandanas) at least in certain public settings, including China, Hong Kong, Spain, Italy (Lombardy region), Russia, and the United States. Besides, they Have also applied social distancing strategies (least 6 feetor $1.8 \mathrm{~m}$ apart) to reduce contact of

\section{Treatment}

\section{General principles of treatment and management}

Symptomatic therapy such as maintaining airway, breathing, circulation, oxygen support (ventilation if required, PO2 $<55 \%$ ), correction of electrolyte imbalance, correct temperature (antipyretic drugs such as Paracetamol) and supporting other affected vital organs are considered as the best to manage patients with different degree of infection [36,37]. The CDC recommends that those who suspect they

\section{Specific treatment}

According to World Health Organization report, as of May 2020, there is no specific treatment for COVID-19 and prevention is the best and therecommended solution for this pandemic since nothing has been found as curative treatment or postulated officially [34]. However, researcherscontinue working on more effective treatments and many vaccine candidates are in development or testing phases. lymphopenia (most common finding), decreased albumin, leukocytosis (WBC count may vary leukopenia), increased lactate dehydrogenase (LDH) levels (due to affinity of cytokines for carolice tissue, hepatic tissue), increasedferritin level (early finding), increased AST \& ALT (aminotransferase),increased total bilirubin, increased Erythrocyte sedimentation rate, increased D-dimer and increased procalcitonin and increased $\mathrm{C}$-reactiveprotein (CRP). These are the main laboratory abnormalities in patients with unfavorable progression of coronavirus disease 2019 (COVID-19).

infected persons with large groups by closing schools and workplaces, restricting travel, and cancelling large public gatherings [12,35]

Those diagnosed with COVID-19 or who believe they may be infected are advised by the CDC to stay home except to get medical care, call ahead before visiting a healthcare provider, wear a face mask before entering the healthcare provider's office and when in any room or vehiclewith another person, cover coughs and sneezes with a tissue, regularly wash hands with soap and water and avoid sharing personal household items. The CDC also recommends that individuals wash hands often with soap and water for at least 20 seconds, especially after going to the toiletor when hands are visibly dirty, before eating and after blowing one's nose, coughing or sneezing. It further recommends using an alcohol- based hand sanitizer with at least $60 \%$ alcohol, but only when soap and water are not readily available. For areas where commercial hand sanitizers are not readily available, the WHO provides two formulationsfor local production. In these formulations, the antimicrobial activity arises from ethanol or isopropanol. Hydrogen peroxide is used to help eliminate bacterial spores in the alcohol; it is "not an active substance forhand antisepsis". Glycerol is added as a humectant $[\mathbf{1 2 , 3 5 ]}$.

carry the virus weara simple face mask. Extracorporeal membrane oxygenation (ECMO) hasbeen used to address the issue of respiratory failure, but its benefits are still under consideration. Personal hygiene with a healthy lifestyle and diet have been recommended to improve immunity. Supportivetreatments may be useful in those with mild symptoms at the early stageof infection $[8,19]$.

For symptoms, paracetamol (acetaminophen) is recommended for first-line use over ibuprofen to alleviate the pain by some medical professionals $[\mathbf{1 4 , 3 8}]$

The U.S. National Institutes of Health guidelines do not recommend anymedication for prevention of COVID-19, before or after exposure to the 
SARS-CoV-2 virus, outside of the setting of a clinical trial. Besides, the WHO and NIH do not oppose the use of non-steroidal antiinflammatorydrugs (NSAIDs) such as ibuprofen for symptoms,

[39] and the FDA says currently there is no evidence that NSAIDs worsen COVID-19 symptoms. Corticosteroids such as methylprednisolone, are not recommended since they have a potential toprolong viral replication [40].

Recently, different drugs are on progressive clinical trials to find the

\section{Ongoing Clinical Trials and its future perspectives}

The COVID-19 Clinical Research Coalition has goals to facilitate rapidreviews of clinical trial proposals by ethics committees and national regulatory agencies, fast-track approvals for the candidate therapeutic compounds, ensure standardized and rapid analysis of emerging efficacyand safety data and facilitate sharing of clinical trial outcomes before publication $[\mathbf{4 3 , 4 4 ]}$.

Different vaccines are tested for their efficacy trials in COVID-19 were concluded from hospitals in China. Globally, there are more than 300 active clinical trials underway as of April 2020. Seven trials were evaluating already approved treatments, including four studies on hydroxychloroquine or chloroquine. Repurposed antiviral drugs make up most of the Chinese research, with nine phase III trials on remdesivir across several countries due to report by the end of April

$[\mathbf{3 8 , 4 5}]$. Other candidates in trials include vasodilators, corticosteroids, immune therapies, lipoic acid, bevacizumab, and recombinant angiotensin- converting enzyme 2 [46].

Moreover, the potential effectiveness of several existing medications arebeing evaluated for the treatment of COVID.19, including remdesivir, chloroquine, hydroxychloroquine, lopinavir/ritonavir, and lopinavir/ritonavir combined with interferon beta $[\mathbf{2 , 4 5}$. Clinical improvement was observed in patients treated with compassionate use remdesivir. Remdesivir inhibits SARS-CoV2 in vitro and Phase III clinical trials are underway in the U.S., China, and Italy $[42,47]$.

Even though, there is no evidence of high-quality evidence exists for theefficacy of chloroquine/hydroxychloroquine treatment of SARS or MERS. Chloroquine inhibits SARS-CoV-2 in vitro with a halfmaximaleffective concentration $\left(\mathrm{EC}_{50}\right)$ in the low micromolar range [48]. A news briefing from China reported chloroquine was

\section{Conclusion}

The COVID-19 pandemic is major public health concern worldwide since 2019 and caused by a novel severe acute respiratory syndrome coronavirus 2 (SARS-CoV-2). Recently, the production and identification of effective drugs or vaccines for prevention and treatmentof the disease remain a great challenge. Even though there are different ongoing clinical trials, production of high-quality vaccine and effective treatment is still a great challenge of the pandemic. Based on recent reports of WHO and CDC, the vaccine for best and effective treatment to this deadly virus. Drugs such as Tocilizumab (IL-6 blockage) and Anakinra (IL-1 blockage) act against effects of Cytokine Storm. Furthermore, antiviral, and antiHIV drugs such as Remidisivr, Lopinavir/ Ritonavir (HIV TE), Favilavir are on the progressof clinical trial. Besides, antimalarials such as Hydrochloroquine also showed promising results [38,39,41]. After a study, remdesivirsuggested to have potential to improve the prognosis of the hospitalizedpatients with severe COVID-19[38,42].

successfully used to treat a series of more than 100 COVID-19 cases resulting in improved radiologic findings, enhanced viral clearance, andreduced disease progression. However, the clinical trial design andoutcomes data have not yet been presented or published for peer review,preventing validation of these claims [38]. This might be since Chloroquine and hydroxychloroquine appear to block viral entry into cells by inhibiting glycosylation of host receptors, proteolytic processing, and endosomal acidification. These agents also have immunomodulatory effects through attenuation of cytokine production and inhibition of autophagy and lysosomal activity in host cells [49].

Even though, there is no available vaccine, various agencies are activelydeveloping vaccine candidates. Previous work on SARS-CoV is being used because both SARS-CoV and SARS-CoV-2 use the ACE2 receptorto enter human cells [26]. Nowadays, scientists are working on developing three vaccination strategies. First, researchers aim to build awhole virus vaccine. The use of such a virus, be it inactive or dead, aimsto elicit a prompt immune response of the human body to a new infectionwith COVID-19. A second strategy, subunit vaccines, aims to create a vaccine that sensitizes the immune system to certain subunits of the virus. In the case of SARS-CoV-2, such research focuses on the $\mathrm{S}$-spikeprotein that helps the virus intrude the ACE2 enzyme receptor. A third strategy is that of the nucleic acid vaccines (DNA or RNA vaccines, a novel technique for creating a vaccination). Experimental vaccines from any of these strategies would have to be tested for safety and efficacy [50]. Besides, antibody-dependent enhancement has been suggested as apotential challenge for vaccine development for SARS-COV-2, but thisis still controversial [51].

COVID-19 is not expected until 2021. Thus, in recent time the best method for managing COVID-19 is to decrease or delay the infection rate of the epidemic stricltly following all the neccassary precautions recommended by WHO. Moreover, this helps to decrease the risk of health services beingoverwhelmed, allowing for better treatment of current cases, and delaying additional cases until effective treatments or a vaccine becomeavailable. 


\section{References}

1. Carlos WG, et al. (2020) Novel wuhan (2019-nCoV) coronavirus. Am J Res Crit Care Medne 201: 7-8.

2. Fesseha H, Fesseha Y (2020) Coronavirus Disease 2019 (COVID- 19) Pandemic-An Update. Adv in Pub Health, Com and Trop Med 1-5.

3. Jernigan DB (2020) Update: Public Health Response to the Coronavirus Disease 2019 Outbreak-United States, February 24, 2020. MMWR. Morbidity and Mortality Weekly Report 69(8): 216-219.

4. Huang C, Wang Y, Li X, Ren L, Zhao J, et al. (2020) Clinical features of patients infected with 2019 novel coronavirus in Wuhan,China. The Lancet 395(10223): 497-506.

5. Richman, D.D., et al. (2016) Clinical virology. John Wiley \& Sons.

6. Zhou C (2020) Evaluating new evidence in the early dynamics of thenovel coronavirus COVID-19 outbreak in Wuhan, China with real time domestic traffic and potential asymptomatic transmissions. MedRxiv.

7. World Health Organization (2020) Coronavirus disease 2019 ( COVID-19): situation report, 115.

8. World Health Organization (2020) Coronavirus disease (COVID19) outbreak.

9. Callaway E (2020) Time to use the p-word? Coronavirus enter dangerous new phase. Nature 579: 12.

10. Chang Le, Yan Y, Wang L (2020) Coronavirus disease 2019: coronaviruses and blood safety. Transfusion medicine reviews 34(2):75-80.

11. Anderson RM, Heesterbeek H, Klinkenberg D, T Déirdre Hollingsworth (2020) How will country-based mitigation measures influence the course of the COVID-19 epidemic? The Lancet 395(10228): 931-934.

12. Center for Disease Control and Prevention (2020) Coronavirus Disease 2019 (COVID-19).

13. Thompson R (2020) Pandemic potential of 2019-nCoV. The LancetInfectious Diseases 20(3): 280.

14. Singhal T (2020) A review of coronavirus disease-2019 (COVID19). Indian J Pediatr 87(4): 281-286.

15. Ahmed SS (2020) The Coronavirus Disease 2019 (COVID-19): A Review. J Adv Med Med Res 32(4): 1-9.

16. Xu Z, Shi L, Wang Y, Zhang J,Huang L, et al. (2020) Pathological findings of COVID-19 associated with acute respiratory distress syndrome. The Lancet Respiratory Medicine 8(4): 420-422.

17. Adhikari SP, Meng S, Wu Y J, Mao Y-P, Rui-Xue Ye, et al. (2020) Epidemiology, causes, clinical manifestation and diagnosis, prevention and control of coronavirus disease (COVID-19) during the early outbreak period: a scoping review. Inf Dis Pov 9: 1-12.
18. Guo YR, Cao QD, Hong ZS, Tan YY, Chen SD, et al. (2020) The origin, transmission and clinical therapies on coronavirus disease 2019 (COVID-19) outbreak-an update on the status. Military Med Res 7: 1-10.

19. Center for Disease Control and Prevention (2020) Severe outcomes among patients with coronavirus disease 2019 (COVID-19)-United States, February 12-March 16, 2020. Morbidity Mortality Weekly Report MMWR 69(12): 343-346.

20. Hanley B, Lucas SB, Youd E, Swift B (2020) Autopsy in suspectedCOVID-19 cases. J Clin Pathol 73: 239-242.

21. Giani M, Seminati D, Lucchini A, Foti G (2020) Exuberant plasmocytosis in bronchoalveolar lavage specimen of the first patient requiring extracorporeal membrane oxygenation for SARS- CoV-2 in Europe. J Thorac Oncol 15(5): e65-e66.

22. Guan WJ, Ni ZY, Hu Y, Liang WH, Ou CQ, et al. (2020) Clinical characteristics of coronavirus disease 2019 in China. New Eng J Med382: 1708-1720.

23. del Rio C, Malani PN (2020) COVID-19-New insights on a rapidly changing epidemic. JAMA 323(14): 1339-1340.

24. Rothan HA, Byrareddy SN (2020) The epidemiology and pathogenesis of coronavirus disease (COVID-19) outbreak. J Autoimmun 102433.

25. Mizumoto K, Kagaya K, Zarebski A, Chowell G (2020) Estimating the asymptomatic proportion of coronavirus disease 2019 (COVID-19) cases on board the Diamond Princess cruise ship, Yokohama, Japan, 2020. Euro Surveill 25(10): 2000180.

26. Cascella M, Rajnik M, Cuomo A, Dulebohn SC, Napoli RD (2020) Features, evaluation and treatment coronavirus (COVID19). In Statpearls [internet], StatPearls Publishing.

27. Carod-Artal F (2020) Neurological complications of coronavirus andCOVID-19. Revista de Neurologia 70(9): 311-322.

28. Hui D, Azhar EI, Madani TA, Ntoumi F, Kock F, et al. (2020) The continuing 2019-nCoV epidemic threat of novel coronaviruses to global health-The latest 2019 novel coronavirus outbreak in Wuhan,China. Int J Infect Dis 91: 264-266.

29. World Health Organization (2020) Laboratory testing for coronavirus disease ( COVID-19) in suspected human cases: interimguidance, 19 March 2020.

30. Zhao S, Lin Q, Ran J, Musa SS, Yang G, et al. (2020) Preliminary estimation of the basic reproduction number of novel coronavirus (2019-nCoV) in China, from 2019 to 2020: A data-driven analysis inthe early phase of the outbreak. Int J Inf Dis 92: 214-217.

31. Fan L, Li D, Xue H, Zhang L, Liu Z, et al. (2020) Progress and prospect on imaging diagnosis of COVID-19. Chin J Acad Radiol 18: $1-10$.

32. Fang Y, Zhang H, Xie J, Lin M, Ying L, et al. (2020) Sensitivity of chest CT for COVID-19: comparison to RT-PCR. Radiol 200432 . 
33. Gong F, Xiong Y, Xiao J, Lin L, Liu X, et al. (2020) China's local governments are combating COVID-19 with unprecedented responses - from a Wenzhou governance perspective. Front Med 14(2): 220-224.

34. World Health Organization (2020) Responding to community spread of COVID-19: interim guidance, 15 May 2020. World Health Organization.

35. World Health Organization (2020) COVID-19 situation update for the WHO European Region- situation report, 60. In WHO Global situation reports, WHO, March, 2020.

36. Morse JS, Lalonde T, Xu S, Liu WR (2020) Learning from the past:possible urgent prevention and treatment options for severe acute respiratory infections caused by 2019-nCoV. Chembiochem 21(5): 730-738.

37. Wang D, Hu Bo, Hu C, Zhu F, Liu X, et al. (2020) Clinical characteristics of 138 hospitalized patients with 2019 novel coronavirus-infected pneumonia in Wuhan, China. JAMA 323(11):1061-1069.

38. Sanders JM, Monogue ML, Jodlowski TZ (2020) Pharmacologic treatments for coronavirus disease 2019 (COVID-19): a review. JAMA 323(18): 1824-1836.

39. National Institutes of Health (2020) COVID-19 Treatment Guidelines". www.nih.gov. Retrieved 21 April 2020.

40. Food and Drug Administration (2020) Coronavirus (COVID-19) Update: FDA Issues Emergency Use Authorization for Potential COVID-19 Treatment". U.S. FDA-Press release.

41. Gao J, Tian Z, Yang Xu (2020) Breakthrough: Chloroquine phosphate has shown apparent efficacy in treatment of COVID19 associated pneumonia in clinical studies. Biosci Trends 14(1):72- 73 .
42. Wang M, Cao R, Zhang L, Yang X, Liu J, et al. (2020) Remdesivir and chloroquine effectively inhibit the recently emerged novel coronavirus (2019-nCoV) in vitro. Cell research 30: 269-271.

43. Zhang L, Liu Y (2020) Potential interventions for novel coronavirusin China: a systemic review. J Med Virol.

44. Dhama K, Sharun K, Tiwari R, Dadar M, Malik YS, et al. (2020) COVID-19, an emerging coronavirus infection: advances and prospects in designing and developing vaccines, immunotherapeutics, and therapeutics. Hum Vaccin Immunother 1- 7.

45. Dong L, Hu S, Gao J, (2020) Discovering drugs to treat coronavirusdisease 2019 (COVID-19). Drug Discov Ther 14: 5860.

46. Shi J, Wen Z, Zhong G, Yang H, Wang C (2020) Susceptibility of ferrets, cats, dogs, and different domestic animals to SARScoronavirus-2. Science. 368(6494): 1016-1020.

47. Li G, De Clercq E (2020) Therapeutic options for the 2019 novel coronavirus (2019-nCoV). Nat Rev Drug Discov 19(3): 149-150.

48. Zhou D, Dai SM, Tong Q (2020) COVID-19: a recommendation to examine the effect of hydroxychloroquine in preventing infection and progression. J Antimicrob Chemother 114.

49. Devaux CA, Rolain JM, Colson P, Raoult D (2020) New insights onthe antiviral effects of chloroquine against coronavirus: what to expect for COVID-19? Int J Antimicrob Agents 55(5): 105938.

50. Chen WH, Strych U, Hotez PJ, Bottazzi ME (2020) The SARSCoV-2 vaccine pipeline: an overview. Curr Trop Med Rep 1-4.

51. Peeples L (2020) News Feature: Avoiding pitfalls in the pursuit of aCOVID-19 vaccine. Proceedings of the National Academy of Sciences 117(15): 8218-8221. 